\title{
Canales y márgenes de comercialización cadena productiva maíz amiláceo (Zea Mays L.) provincia de Chachapoyas
}

\section{Channels and margins of commercialization productive chain starchy corn (Zea Mays L.) province of Chachapoyas}

\author{
Robert Javier Cruzalegui Fernández ${ }^{1}$, Euclides Walter Luque Chuquija ${ }^{2}$
}

\begin{abstract}
RESUMEN
Se analizó los canales y márgenes de comercialización en la cadena productiva del maíz amiláceo (Zea Mays l.) en la provincia de Chachapoyas, entrevistando a 71 productores de maíz amiláceo, 8 comerciantes mayoristas y 14 comerciantes detallistas identificados; distribuidos en los mercados de Yerbabuena, Asunción, Pipus y Chachapoyas. Se utilizó el tipo de investigación descriptivo, con un estudio por muestreo aleatorio, utilizándose para el análisis de datos la prueba de Kruskal-Wallis, debido a que no existió normalidad significativa en los datos para realizar una comparación de medias. Los canales de comercialización identificados fueron dos, el primero: productor de maíz amiláceo, comerciante mayorista, comerciante detallista y consumidor final; el segundo: productor de maíz amiláceo, comerciante detallista y consumidor final. Además, se determinó la rentabilidad en los canales de comercialización, obteniendo mejor rentabilidad el comerciante detallista con un $21.98 \%$ muy superior a los demás eslabones de la cadena productiva. La participación del productor en la cadena productiva es del $62.76 \%$ y la de los intermediarios del $37.24 \%$. Finalmente, a un intervalo de confianza del $99 \%$ se rechaza la hipótesis nula (H0) y se acepta la hipótesis alternativa (Hi) y se concluye que los canales de comercialización del maíz amiláceo no se diferencian significativamente con los márgenes de comercialización del maíz amiláceo.
\end{abstract}

Palabras clave: Canales, márgenes, comercialización, cadena.

\section{ABSTRACT}

Marketing channels and margins were analyzed in the production chain of starchy corn (Zea Mays $l$.) In the province of Chachapoyas, interviewing 71 producers of starchy corn, 8 wholesalers and 14 identified retailers; distributed in the markets of Yerbabuena, Asunción, Pipus and Chachapoyas. The type of descriptive research was used, with a random sampling study, using the Kruskal-Wallis test for data analysis, because there was no significant normality in the data to make a comparison of means. The commercialization channels identified were two, the first: amylocean corn producer, wholesaler, retailer and final consumer; the second: amylocean corn producer, retailer and final consumer. In addition, the profitability was determined in the marketing channels, with the retail merchant obtaining a better profitability with a $21.98 \%$ much higher than the other links in the production chain. The producer's participation in the productive chain is $62.76 \%$ and that of the intermediaries of $37.24 \%$. Finally, at a confidence interval of $99 \%$, the null hypothesis (H0) is rejected and the alternative hypothesis (Hi) is accepted and it is concluded that the marketing channels of starchy corn do not differ significantly with the marketing margins of starchy corn.

Keywords: Channels, margins, marketing, chain.

\footnotetext{
${ }^{1}$ Ingeniero Agroindustrial, Profesor Auxiliar Tiempo Completo de la Facultad de Ingeniería y Ciencias Agrarias de la Universidad Nacional Toribio Rodríguez de Mendoza de Amazonas. Correo electrónico: robert.cruzalegui@untrm.edu.pe

${ }^{2}$ Antropólogo, Profesor Principal Tiempo Completo de la Facultad de Derecho y Ciencias Políticas de la Universidad Nacional Toribio Rodríguez de Mendoza deAmazonas. Correo electrónico: euclidesluque@hotmail.com
} 


\section{INTRODUCCIÓN}

El maíz amiláceo, es un cereal que en los últimos 50 años ha mostrado una tasa de crecimiento negativa en su consumo per cápita, pasando de $18 \mathrm{~kg} /$ habitante en el año 1,950 a $8.6 \mathrm{~kg} /$ habitantes en el año 2007 , esto debido a que la población andina había iniciado la migración hacia la costa, abandonando sus hábitos de consuno tradicionales. Sin embargo, al 2011 esta tendencia registra un incremento de consumo de 9.7 $\mathrm{kg}$ por habitante (MINAG, 2011). Además, es importante por ser parte como insumo en la ganadería vacuna y en la crianza de animales menores; actividades económicas que se desarrollan en esta región con producciones importantes (INEI, 2012). En el año 2012 la provincia de Chachapoyas contaba con aproximadamente 1,936 hectáreas instaladas de maíz amiláceo (Zea Mays 1.) con rendimientos promedios que van desde los 800 $\mathrm{kg} / \mathrm{Ha}$ y son comercializados mediante diferentes canales de comercialización tanto a nivel local, regional y nacional (MINAG, 2012).

En cuanto a la comercialización de productos agrícolas en el Perú esta se encuentra regulada por la ley del libre mercado; en consecuencia, esta es desordenada y no cuenta con políticas agrarias orientadas a reducir los efectos de dicho desorden (Escobal, 1994). Uno de los resultados de este modelo es que los productos agropecuarios son comercializados, en su mayoría, por un acopiador o intermediario quien compra la cosecha en chacra o en mercados locales, fijando el precio de acuerdo a condiciones solicitadas por el mayorista, para posteriormente trasladarla a mercados mayoristas. En estos mercados el mayorista, después de clasificar, seleccionar y/ o envasar, vende el producto al minorista quien a su vez lo lleva a mercados y expendios locales para su venta al consumidor final (García et al., 2012).

Como es entendible el precio de un producto agropecuario puede dividirse en sus componentes: precio al productor y precio de la comercialización del campo al consumidor final. La parte que cubre la comercialización también se denomina margen de comercialización y corresponde a la diferencia entre lo que paga el consumidor y lo que recibe el productor en campo (Schwentesius \& Gómez, 2004). Este incluye todos los gastos que se efectúan para agregarle valor al producto, para almacenarlo, acondicionarlo, transportarlo y para ofrecerlo al consumidor y, además, incluye las ganancias de los agentes de transformación, almacenamiento, distribución y comercialización; en este sentido el consumidor está pagando dos precios: el precio del productor agropecuario y el precio o margen de comercialización. Estos precios reflejan el costo de producción en campo y el costo del servicio de transformación y comercialización (incluye costos y ganancias de transporte, almacenamiento, transformación y distribución (Schwentesius \& Gómez, 2004).

En este recorrido descrito anteriormente que realiza el producto el precio por unidad se incrementa, por efecto de los costos de comercialización, a fin de cubrir los riesgos del mercadeo y generar una retribución o beneficio neto a los participantes en el proceso de distribución de la producción agrícola desde el productor hasta el detallista en los mercados de comercialización de dicho producto (Shepherd, 1995).

El objetivo de investigación fue analizar los canales y márgenes de comercialización de la cadena productiva del maíz amiláceo en la provincia de Chachapoyas, región Amazonas.

\section{MATERIAL Y MÉTODO}

\section{Ubicación del área de estudio:}

El área de estudio se desarrolló en los mercados de Yerbabuena, Asunción, Pipus y Chachapoyas, ubicadas en la provincia de Chachapoyas.

\section{Diseño de la investigación:}

Se aplicó un diseño no experimental de carácter transversal o transeccional - correlacional, que tiene como objetivo describir relaciones entre dos o más variables en un momento determinado, con muestreo probabilístico para la aplicación de las encuestas, se elaboró de manera que permita adquirir la información suficiente y necesaria en la provincia de Chachapoyas para tener conocimiento sobre los canales y márgenes de comercialización de la cadena productiva del maíz amiláceo.

$$
\mathbf{X}_{1} \rightarrow \mathbf{Y}_{1}
$$

Dónde: $X_{1}$ : Canales de comercialización $Y_{1}$ : Márgenes de comercialización

\section{Población y muestra}

Población: La población está conformada por 2,372 productores de maíz amiláceo que cuentan con parcelas instaladas en el ámbito de la provincia y que comercializan su producción en los mercados más representativos de la provincia de Chachapoyas, según el sistema de consulta del IV Censo Nacional Agropecuario 2012.

Muestra: Para determinar la muestra de la 
investigación se tomó como base la población de productores de maíz amiláceo $(2,372)$ a través de la fórmula estadística de muestreo se determinó el tamaño de la misma.

$$
n=\frac{N * Z_{\propto}^{2} * p * q}{d^{2} *(N-1)+Z_{\propto}^{2} * p * q}
$$

$$
\begin{aligned}
& \text { Donde: } \\
& n \quad \text { : Tamaño de la muestra. } \\
& N \quad \text { : Total de la población. } \\
& Z_{\square} \quad \text { : Nivel de confianza. } \\
& p \quad \text { : Variabilidad positiva } \\
& q \quad: \text { Variabilidad negativa }(1-p) \\
& \text { d } \quad \text { : Grado de error. } \\
& n=\frac{2,372 * 1.96^{2} * 0.05 * 0.95}{0.05^{2} *(2,372-1)+1.96^{2} * 0.05 * 0.95} \\
& n=71
\end{aligned}
$$

La muestra de productores de maíz amiláceo se conformó por 71 productores. En cuanto a la muestra de comerciantes, debido a su menor número en relación a los productores, se aplicó la encuesta a la totalidad de los agentes identificados en los mercados antes mencionados.

\section{Método de recolección de datos}

El método inició con la formulación del problema de investigación, identificación de la información necesaria para resolver el problema, elaboración de instrumentos para la recolección de datos, identificación de la población de estudio, establecimiento de un método de muestreo, diseño del proceso para la obtención de datos, recopilación de datos de cada mercado, análisis de datos y preparación del informe final.

\section{Técnicas}

Las técnicas utilizadas para la recolección de datos de la presente investigación fueron: documental y encuesta aplicada en campo.

\section{Instrumentos}

Técnica documental: Recopilación de información para enunciar las teorías que sustentan el estudio por medio de fichas bibliográficas.

Técnica de campo: Análisis de los márgenes de comercialización en cadenas productivas, según la metodología recomendada por Salazar y Heyden, (2004), la cual permitirá la observación en contacto directo con el objeto de estudio y el acopio de testimonios de los productores del cultivo de maíz amiláceo a través de fichas de observación y cuestionario de encuesta, una para productores otra para comerciantes mayoristas y finalmente otra para detallistas.

\section{RESULTADOS}
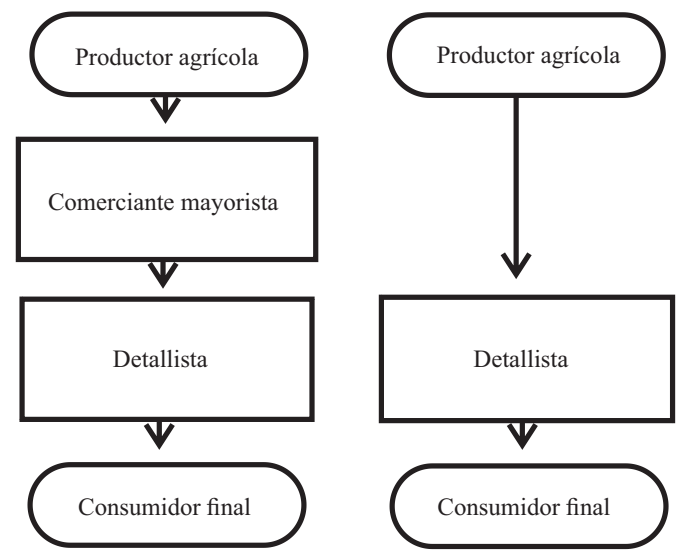

Figura 1. Canales de comercialización del maíz amiláceo en la provincia de Chachapoyas

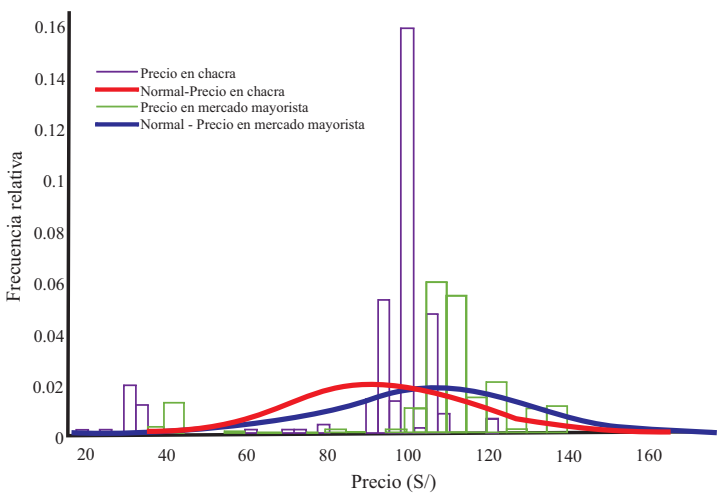

Figura 2. Precio en chacra vs precio en mercado mayorista

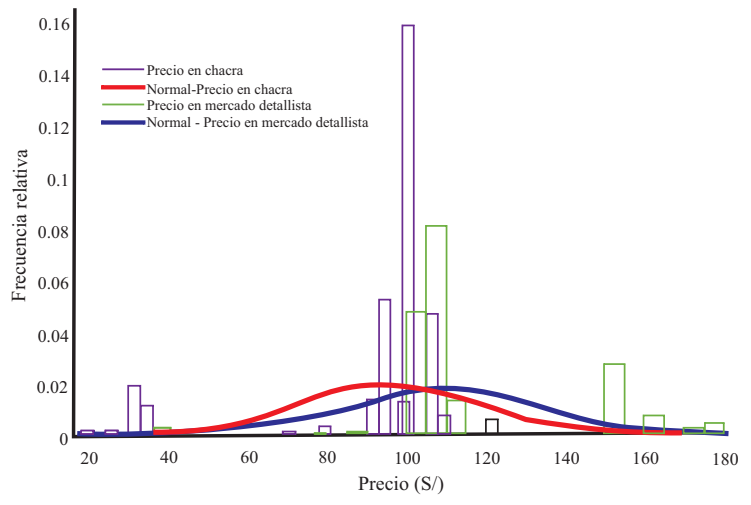

Figura 3. Precio en chacra vs precio en mercado detallista 


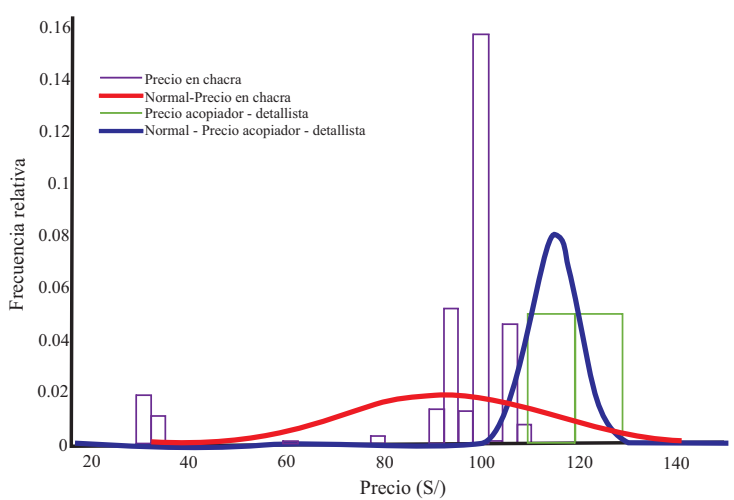

Figura 4. Precio en chacra vs precio acopiador detallista

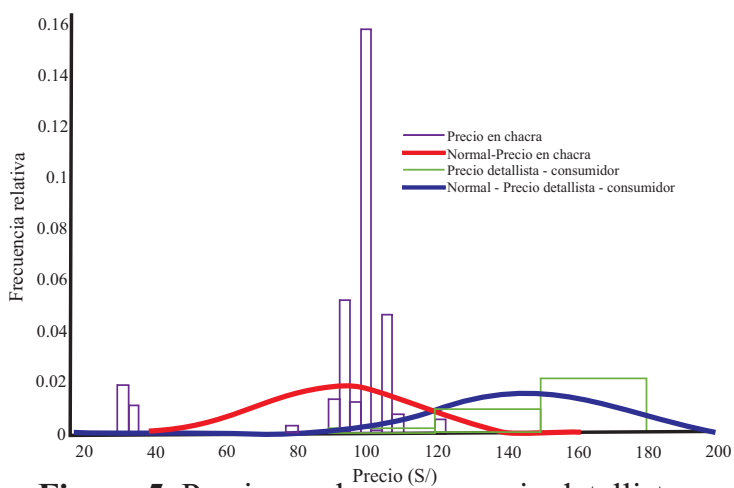

Figura 5. Precio en chacra vs precio detallista consumidor final

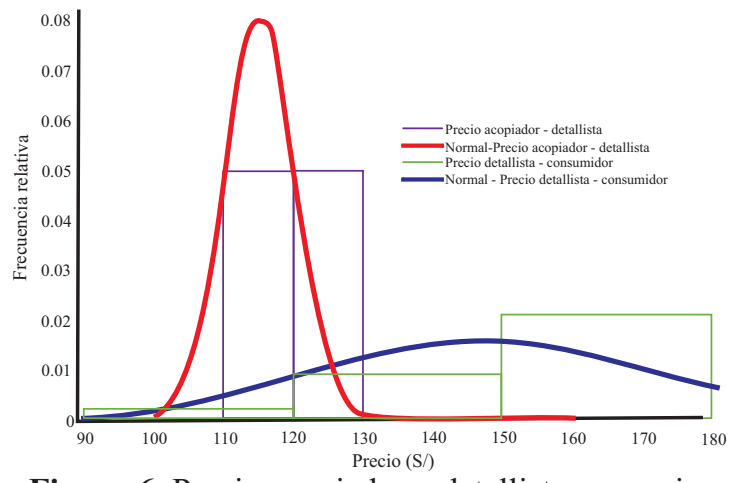

Figura 6. Precio acopiador - detallista vs precio detallista - consumidor

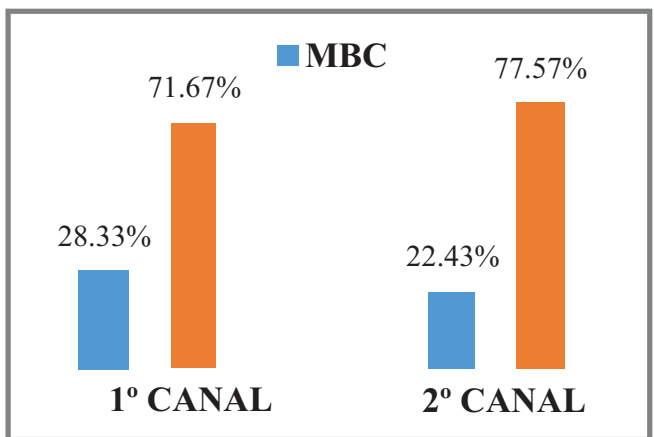

Figura 7. Rentabilidad de los canales de comercialización 1 y 2

\section{DISCUSIÓN}

La cadena de comercialización del maíz amiláceo en la provincia de Chachapoyas tiene dos canales de comercialización claramente identificados (Figura 1); la primera se conforma por los eslabones productor agrícola, comerciante mayorista, detallista y consumidor final en cuanto a la segunda por los eslabones productor agrícola, detallista y consumidor final. En la identificación de estos canales de comercialización no se ha considerado la comercialización del maíz amiláceo realizada en otras regiones como San Martin y Cajamarca (Jaén), esto difiere de lo reportada por Andrade, (2014), que concluye que la cadena de valor del maíz (Zea mays) en la región 4 provincia de Manabí está conformada por productor, intermediario, industria, distribuidor, consumidor final y que estas cadenas de comercialización se caracterizan por el rol que juega el gobierno al proteger al productor fijando los precios y cuotas de producción.

El margen bruto de comercialización del maíz amiláceo en el eslabón de la cadena precio chacra y precio mayorista (figura 2) tiene un valor de $8.91 \% \mathrm{el}$ cual se encuentra en el rango reportado por Vidal, (2014) de 7 a $13 \%$ para pequeños y grandes productores respectivamente. Además, los márgenes brutos de comercialización del primer y segundo del maíz amiláceo en la provincia de Chachapoyas oscilan entre 28.33 y $22.43 \%$ respectivamente, enmarcados en los márgenes de comercialización en condiciones de baja tecnología que van de 21 a $42 \%$ sobre las ventas según lo precisado por Vidal, (2014).

El margen bruto de comercialización del segundo canal de comercialización de la cadena productiva del maíz amiláceo en la provincia de Chachapoyas es de $22.43 \%$ (Figura 7), lo que significa que el $77.57 \%$ del precio que paga el consumidor final lo percibe el productor agrícola, mientras que el $22.43 \%$ lo perciben los intermediarios, cantidades semejantes a los reportados por Hernández et al., (2011), que calculó los márgenes de comercialización en otro producto agropecuario, piñón con cascara, encontrando que los intermediarios se apropian del $22.12 \%$ y los productores el $77.88 \%$ del precio que paga el consumidor final. Asimismo, Espinosa y Rivera (2008), obtuvo $31.7 \%$ de margen bruto de comercialización para los acopiadores que comercializan leche cruda, lo que significa que 68.3 $\%$ del precio pagado por el consumidor final lo percibe el productor de leche cruda y el $31.7 \%$ se apropia el intermediario lo cual es concordante con lo determinado en esta investigación.

En cuanto al rendimiento del maíz amiláceo, en la 
provincia de Chachapoyas, su promedio es de 937.50 $\mathrm{Kg} / \mathrm{ha}$; este valor se encuentra muy por debajo del promedio nacional $(1,606 \mathrm{Kg} / \mathrm{ha})$ agravándose en el caso del promedio regional $(845 \mathrm{Kg} / \mathrm{ha})$ según lo reportado por Huamanchumo (2013).

Además, se determinó que el $47.05 \%$ del total de la producción del maíz amiláceo en la provincia de Chachapoyas es destinado a la comercialización, principalmente en grano seco, mientras que Colombia importa el $65.8 \%$ de maíz en general según lo concluido por Aza, (2014) por lo que podría ser un mercado potencial para la exportación el maíz amiláceo en la provincia de Chachapoyas.

Del análisis estadístico, a un intervalo de confianza del $99 \%$, se acepta la hipótesis alternativa (Hi) y se rechaza la hipótesis nula (H0) concluyendo que los canales de comercialización del maíz amiláceo no se diferencian significativamente con los márgenes de comercialización del maíz amiláceo, diferente a lo reportado por Viejo (2013), en su estudio con productores de caña de azúcar; que concluye que su hipótesis planteada se ha comprobado, que controlando la medición de los niveles de inversión en la cadena de valor del cultivo de la caña de azúcar permitirá realizar un mejoramiento en los márgenes de rentabilidad de los productores de caña y sus familias.

\section{CONCLUSIÓN}

La investigación permitió conocer los canales y márgenes de comercialización del maíz amiláceo en la provincia de Chachapoyas región Amazonas; centrando esta labor en productores, comerciantes mayoristas y detallistas exclusivamente de la provincia de Chachapoyas.

De acuerdo a los resultados se identificaron dos canales de comercialización del maíz amiláceo en la provincia de Chachapoyas que utiliza el agricultor. El primer canal de comercialización comprende los eslabones de productor agrícola, comerciante mayorista, comerciante detallista y consumidor final; mientras que el segundo canal de comercialización se encuentra comprendido por los eslabones de productor agrícola, comerciante detallista y consumidor final.

El margen bruto de comercialización del primer canal de comercialización es del $28.33 \%$, mientras que la participación del productor en el mencionado canal de comercialización es del $71.67 \%$; en el segundo canal de comercialización con un margen bruto de comercialización de $22.43 \%$.
El productor agrícola percibe mayor margen de comercialización en el segundo canal de comercialización de $14.81 \%$ por sobre el $8.91 \%$ del primer canal.

El comerciante detallista es el más beneficiado en los dos canales de comercialización con márgenes brutos de comercialización entre 21.98 y $22.43 \%$ para el primero y segundo canal respectivamente.

Mediante la aplicación de la prueba de KruskalWallis, se concluye que a un intervalo de confianza del $99 \%$ se concluye que los canales de comercialización del maíz amiláceo no se diferencian significativamente con los márgenes de comercialización del maíz amiláceo, por lo que se rechaza la hipótesis nula y se acepta la hipótesis alternativa.

Mediante la aplicación de la prueba de la Mediana de Mood, cuyo objetivo es comparar las medianas de dos muestras se contrasta la hipótesis y se confirma que los canales de comercialización no se diferencian significativamente con los márgenes de comercialización en la cadena productiva del maíz amiláceo de la provincia de Chachapoyas.

\section{REFERENCIAS BIBLIOGRÁFICAS}

Aza, M. (2014). Estrategia de comercialización de maíz suave amarillo en Carchi - Ecuador y la demanda efectiva en el Valle del Cauca Colombia. Tesis de pregrado. Universidad Politécnica Estatal del Carchi, Ecuador.

Escobal, J. (1994). Comercialización agrícola en el Perú. Grupo Análisis Para El Desarrollo. Prim. Ed. Lima.

Espinosa, A. (2009). La competitividad del sistema agroalimentario localizado productor de quesos tradicionales. Tesis Doctoral. Universidad Autónoma del Estado de México. México.

Espinosa, O., y Rivera, H. (2008). Los canales y márgenes de comercialización de la leche cruda producida en sistema familiar. Vet. México Vol 39 Num 1 enero - marzo 2008 Pp 1 - 16.

García, M., Hernández, A., y Samaniego, T. (2012). Estrategias de comercialización. Contrib. Econ. Univ. Autónoma Tamaulipas México.

Hernández, M., Islas, G., y Guerra, C. (2011). Márgenes de comercialización del piñón (Pinus cembroides subesp. orizabensis) en Tlaxcala, México. Rev. Mex. Cienc. Agríc. Vól 1 Núm 2 marzo - Abril Pp 265 279. 
Huamanchumo, C. (2013). La cadena de valor de maíz en el Perú: diagnóstico del estado actual, tendencias y perspectivas. (IICA, Lima (Perú)).

INEI (2012). Perú: Consumo per cápita de los principales alimentos 2008 - 2009 (Lima, Perú).

Martínez, C., Cotera, R., and Kido, C. (2013). Canales y márgenes de comercialización de productos lácteos en Dobladero, Veracruz, México.

Martínez, C., Cotera, R., Arceo, M., Damien, F., y Kido, C. (2015). Agentes y márgenes de comercialización del ganado bovino para abasto en Loma Bonita, Oaxaca.

MINAG (2012). Maíz Amiláceo: Principales Aspectos de la Cadena Productiva (Lima, Perú).

Molina, J., y Bello, S. (2011). Estrategias de comercialización de piña (Ananas sativus schult)y pitahaya (Hylocereus undatos Haw) y sus derivados: Vinos y mermeladas, para la cooperativa agroindustrial de productores de San Ignacio (COAPROSI), en la concepción, Masaya 2008-2011. bachelor. Universidad Nacional Agraria, UNA.

Nicholson, W. (2008). Teoría microeconómica. Principios básicos y ampliaciones. Cengage Learn. Ed. SA México Mex.

Porter, M. (2004). Estrategia competitiva. México Trigésima Cuarta Reimpr. Ed CECSA México.

Quispe, N. (2013). Análisis de la Cadena de Valor de la Papa Nativa en los distritos de Huayana y Pomacocha-Provincia de Andahuaylas - Apurímac. Tesis de maestría. Pontificia Universidad Católica del Perú.

Rebollar, R., Hernández, M., García, S., García, M., Torres, H., Bórquez, G., y Mejía, H. (2007). Canales y márgenes de comercialización de caprinos en Tejupilco y Amatepec, estado de México.

Rebollar, R., Hernández, M., García, M., Albarrán, P., Rojo, R., y Gonzáles, R. (2011). Canales y márgenes de comercialización del queso añejo en Zacazonapan, México.

Rivera, N. (2011). La Caña de Azúcar y sus Derivados en la Huasteca San Luís Potosí México. Diálogos Rev. Electrónica 11.

Salazar, M., y Heyden, D. (2004). Metodología de análisis de cadenas productivas con equidad para la promoción del desarrollo local. Lima - Perú.

Sampieri, R., Collado, C.F., y Lucio, P.B. (2010). Metodología de la Investigación. México El Comer. SA.
Samuelson, P.A., y Nordhaus, W.D. (2010). Economía. Editor. Mc Graw Hill Decimonovena Ed. México - México.

Schwentesius, R., y Gómez, M. (2004). Márgenes y costos de comercialización: Aspectos conceptuales. Chapingo México.

Shepherd, A. (1995). Guía para el cálculo de los costos de comercialización (FAO, Roma).

Stanton, W., Etzel, M., y Walker, B. (2007). Fundamentos de marketing. Editor. Mc Graw Hill Decimocuarta Ed. México México.

Vidal, M. (2014). Características de la cadena productiva de maíz en la región Lambayeque. Tesis de pregrado. Universidad Católica Santo Toribio de Mogrovejo.

Viejo, K. (2013). Estudio de la cadena de valor de la caña de azúcar (saccharum officinarum) en el recinto tres postes de la provincia del Guayas. Tesis de pregrado. Universidad de Ecuador. 